\title{
Placental pathology concerning sudden foetal demise in SARS-CoV-2 positive asymptomatic pregnant female
}

\author{
Kamil Biringera , Jana Sivakovaa, Juraj Marcinek ${ }^{\mathrm{b}}$, Terezia Pribulova ${ }^{\mathrm{a}}$, Tomas Rokos ${ }^{\mathrm{a}}$, Erik Kozubika, Erik Kudela ${ }^{\mathrm{a}}$, Lukas Plank
}

\begin{abstract}
Aims. Coronavirus disease 2019 is responsible for a worldwide increase in morbidity and mortality. The relationship of this infection to mother-to-child vertical transmission has not been elucidated yet. However, recent reports indicate a foetal death rate of up to $3 \%$.

Methods. We report a case of sudden pre-term foetal demise in a woman positive for SARS-CoV-2 but asymptomatic, with physiological course of pregnancy.

Results. One of the possible explanations of sudden foetal death may be acute placental insufficiency caused by a SARS-CoV-2 placental infection or the development of foetal inflammatory response syndrome (FIRS).

Conclusion. Considering the potential risk of foetal demise, questions remain regarding foetal monitoring and the timing of labour and delivery in the second and third trimesters, particularly in asymptomatic or mild maternal SARSCoV-2 infection. A relevant multidisciplinary team must also be aware of these risks associated with possibly fatal consequences.
\end{abstract}

Key words: SARS-CoV-2, COVID-19, placenta, foetal demise

Received: April 15, 2021; Revised: June 25, 2021; Accepted: June 28, 2021; Available online: July 20, 2021

https://doi.org/10.5507/bp.2021.044

(c) 2021 The Authors; https://creativecommons.org/licenses/by/4.0/

${ }^{a}$ Department of Gynaecology and Obstetrics, Jessenius Faculty of Medicine in Martin, Comenius University in Bratislava, Slovak Republic ${ }^{b}$ Department of Pathological Anatomy, Jessenius Faculty of Medicine in Martin, Comenius University in Bratislava, Slovak Republic Corresponding author: Erik Kudela, e-mail: kudela.erik@gmail.com

\section{INTRODUCTION}

Coronavirus disease 2019 (COVID-19) is caused by a novel coronavirus, now called severe acute respiratory syndrome coronavirus 2 (SARS-CoV-2). The vast majority of those infected are asymptomatic or have only mild to moderate cold- or flu-like symptoms. Cough, fever, and dyspnoea are other relevant symptoms. Serious complications, such as pneumonia and severe hypoxia, have been reported in patients with COVID-19, particularly in the elderly, immunosuppressed patients, and those with chronic diseases (e.g., diabetes, cancer, and chronic lung disease). In association with the occurrence of new virus mutations, a tendency for an increase in SARS-CoV-2 positivity in the younger population has occurred. However, these symptoms and complications may also occur in pregnant women. The relationship of this infection to mother-tochild vertical transmission has not been elucidated yet. However, recent reports of the Centers for Disease Control and Prevention indicate a foetal death rate of $2.2 \%-3 \%$. (ref. ${ }^{1,2}$ ) It seems that one of the main causes of intrauterine foetal death may be morphological and functional changes in placental tissue ${ }^{3}$. We report a case of foetal demise in a woman positive for SARS-CoV-2 but asymptomatic.

\section{CASE REPORT}

A 21-year-old secundigravida primipara with controlled hypothyroidism in her personal history and normal course of pregnancy, together with negative prenatal screening (including ultrasound, toxoplasmosis, rubella, cytomegalovirus, herpes simplex, and human immunodeficiency virus), tested positive for SARS-CoV-2 at 29+2 weeks of gestation. She had no COVID-19 symptoms, and she was followed by the outpatient department. The results of cardiotocography (CTG) and ultrasound (US) at $30+4$ weeks of gestation $\left(9^{\text {th }}\right.$ day of SARS-CoV-2 positivity) were normal. CTG revealed a heart rate of $150 \mathrm{bpm}$, variability of undulatory type, spontaneous accelerations, no decelerations, and no contractions (Fig. 1). US revealed BPD $79 \mathrm{~mm}$, HC $306 \mathrm{~mm}$, AC 253 mm, FL 62 mm, EFW 1,659 g +/- 242 g (51.8\% Williams), normal placental appearance, normal Doppler flow parameters (UA-PI 0.73, MCA-PI 1.58, and CPR 2.16 ( $52^{\text {nd }}$ percentile)), cervical length $30 \mathrm{~mm}$, and no cervical funnelling. The patient was admitted to the hospital 4 days later at $31+1$ weeks of gestation with mild vaginal bleeding, no presence of foetal movements, and no signs of rupture of membranes. Maternal vital signs were normal (BP 138/84 mmHg, HR 88 bpm, BT $36.0^{\circ} \mathrm{C}$, and SpO2 99\%). US confirmed foetal asystole. Uncomplicated induced labour and delivery were finished within $3 \mathrm{~h}$. A female foetus with a weight of $1,650 \mathrm{~g}$ and length of $41 \mathrm{~cm}$ was delivered with no morphological abnormalities. Maternal laboratory parameters were as follows: predelivery - WBC $8.9 \mathrm{x}$ $10^{9} / \mathrm{L}, \mathrm{Hb} 13.7 \mathrm{~g} / \mathrm{L}$, Hct 0.42, PLT $134 \times 10^{9} / \mathrm{L}$, CRP 33.7 $\mathrm{mg} / \mathrm{L}$ and postdelivery - WBC $9.8 \times 10^{9} / \mathrm{L}, \mathrm{Hb} 11.3 \mathrm{~g} / \mathrm{L}$, Hct 0.34, PLT $124 \times 10^{9} / \mathrm{L}$, and RT-PCR anti-SARS-CoV-2 positive, respectively. The patient was discharged home the same day with a normal postpartum course. 


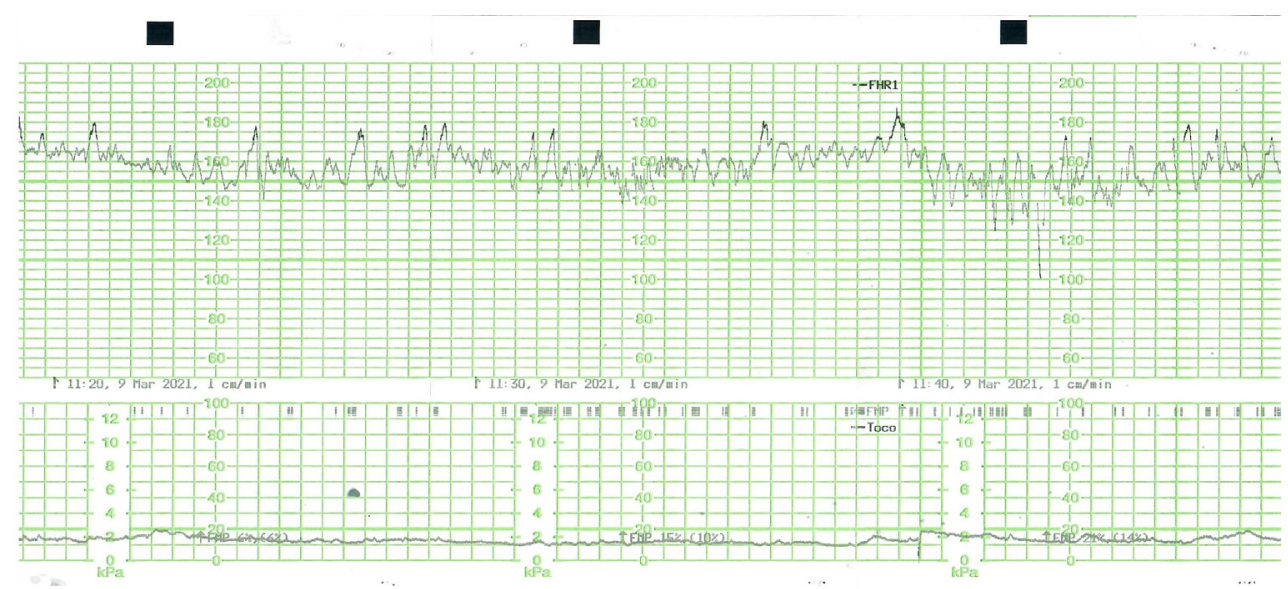

Fig. 1. Cardiotocography at 29+2 weeks of gestation.
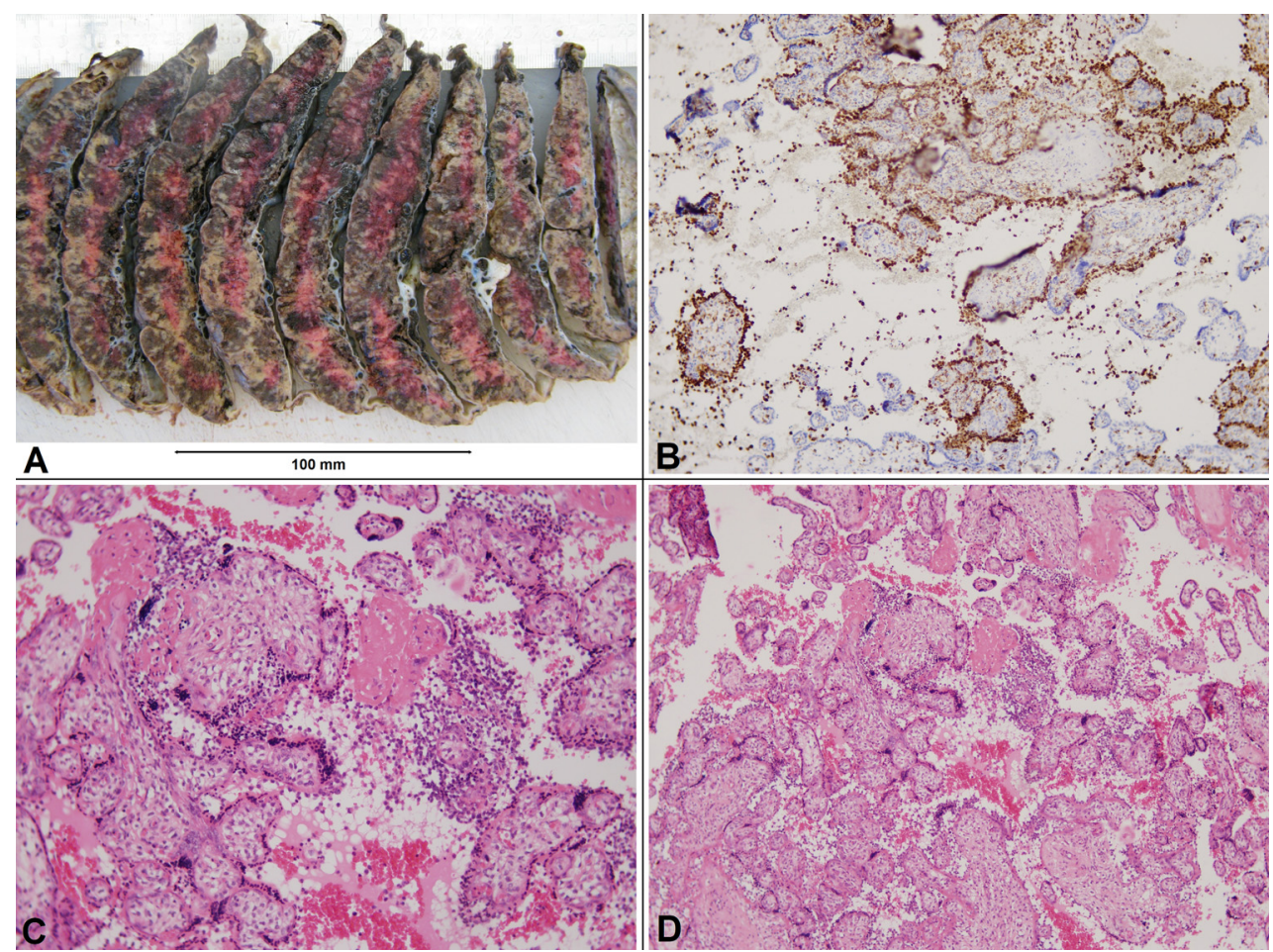

Fig. 2. Histopathological assessment of placenta.

A - macroscopic appearance of the placenta; B - immunohistochemistry for histiocytic intervillositis (CD68 antibody stain, $\mathrm{x} 100$ ); C and D - remnants of hypo- and avascularised mature chorionic villi enveloped by florid, predominantly histiocytic inflammation with neutrophils, finally destroying the trophoblast, without spread into the villous stroma (Haematoxylin and eosin, x200 and x100).

Histological results confirmed a placenta with CD68 positivity (a predominantly intervillous histiocytic inflammatory reaction with dispersed neutrophils and massive fibrin deposits), "maternal floor infarctions," mature chorionic villi, reduced and/or missing placental vascularity, absence of the trophoblastic superficial layer, and no signs of amnionitis or funisitis. Foetal organs had an appropriate degree of maturation with no morphological abnormalities (Fig. 2).

\section{DISCUSSION}

Many epidemiological studies have linked SARSCoV-2 infection to an increase in overall morbidity and mortality worldwide. This infection is asymptomatic or with only mild flu-like symptoms in most cases. A large proportion of those affected are completely cured, but some individuals may develop post-COVID syndrome. The main organs affected are the lungs whose injury can lead to respiratory insufficiency, failure, and even death. 
The main biological manifestations of SARS-CoV-2 infection are pneumocyte damage, alveolar haemorrhage, macrophage clustering, intra-alveolar fibrin exudate, interstitial fibrosis, and inflammatory infiltrate. Major vascular findings, including thrombi in medium- and large-calibre vessels, platelet microthrombi, and fibrin microthrombi, have also been detected ${ }^{4-5}$.

Unlike postpartum life, where the role of respiration is taken over by the lungs, the exchange of foetal respiratory gases takes place in the placenta. It is also a natural relative barrier between mother and foetus. Therefore, it may play a significant role in the vertical spread of SARSCoV-2 infection from mother to foetus.

Previous testing indicated a placental infection with SARS-CoV-2. Trophoblastic cells, which are in direct contact with the maternal blood in the intervillous space, showed marked expression of angiotensin-converting enzyme 2 (ACE2) throughout pregnancy. ACE2, as a receptor, mediates the entry of SARS-CoV-2 into human cells, supporting the fact that the virus can infect the placenta via a receptor-mediated mechanism. As a result, it can cross the placental barrier towards the foetus. However, such vertical transmission needs further investigation ${ }^{6,7}$.

In our case, we found a placenta with CD68 positivity (a predominantly intervillous histiocytic inflammatory reaction with dispersed neutrophils and massive fibrin deposits), "maternal floor infarctions," mature chorionic villi, reduced and/or missing placental vascularity, absence of the trophoblastic superficial layer, and no signs of amnionitis or funisitis. Foetal organs had an appropriate degree of maturation with no morphological abnormalities. Our findings are in agreement with the results published by Menter et al. They documented different time points regarding SARS-CoV-2 infection. In acute COVID-19, prominent lymphohistiocytic villitis may occur and may potentially be attributable to SARS-CoV-2 infection of the placenta. Furthermore, they found histopathological signs of maternal and foetal malperfusion, and they hypothesize this may have a relationship with an altered coagulative or microangiopathic state induced by SARS-CoV-2. (ref. $^{8}$ )

Acute or chronic placental insufficiency induced by SARS-CoV-2 infection can be responsible for subsequent miscarriage or foetal growth restriction ${ }^{7,9}$. Similarly, such a condition could result in foetal loss in the higher stages of pregnancy.

In addition to the morphological and functional damage to the placenta itself, the foetus may be endangered by other mechanisms. Some authors hypothesize that systemic inflammation occurs in response to maternal viral infection in the absence of vertical transmission of the virus. It will be important to consider the virus as a nidus for foetal inflammatory response syndrome (FIRS) and resulting morbidity ${ }^{10}$. A higher rate of perinatal foetal distress and admission to the neonatal intensive care unit has been identified in neonates born to mothers infected with SARS-CoV-2. (ref. ${ }^{11,12}$ ) Neonates affected by FIRS have multiorgan system involvement and higher morbidity after adjustment for gestational age. McCarty et al. hypothesize that FIRS is secondary to exposure to mater- nal SARS-CoV-2 infection in utero and can occur in the absence of proven vertical transmission ${ }^{10}$. Also, a novel childhood hyperinflammatory syndrome presumed to be associated with and subsequent to SARS-CoV-2 infection. The following multisystem inflammatory disorder in children (MIS-C) most commonly affects young children and is characterized by persistent fever, systemic hyperinflammation, and multisystem organ dysfunction ${ }^{13}$. Therefore, we can assume, FIRS secondary to maternal SARS-CoV-2 infection could be a cause of foetal and neonatal morbidity and mortality.

On the other hand, in a large, single-institution cohort study ( 252 tested positive for SARS-CoV-2), infection during pregnancy was not associated with adverse pregnancy outcomes. Neonatal infection may be as high as $3 \%$, but the paradox is that it may occur predominantly among asymptomatic or mildly symptomatic women ${ }^{14}$.

In our case, there was a sudden death of the foetus, which did not show signs of chronic placental insufficiency. Its birth weight and length were appropriate for gestational age, and diagnostic methods before determining foetal death showed no pathology of the foetus, placenta, or umbilical cord. Foetal demise occurred within 4 days after the last examination, despite an asymptomatic maternal infection. Postpartum examinations showed only placental injury. Therefore, we hypothesize that one of the possible explanations may be sudden foetal death due to acute placental insufficiency caused by SARS-CoV-2 placental infection or the development of FIRS with fulminant foetal death.

The strengths of this case report include consistent prenatal screening and diagnosis of foetal well-being, reliable methods confirming maternal SARS-CoV-2 infection, and histopathological assessment by experienced paediatric pathologists. The main limitation is the single case with no foetal and placental SARS-CoV-2 RT-PCR testing.

\section{CONCLUSION}

Considering the potential risk of foetal death, questions remain regarding the manner and frequency of foetal monitoring, the decision to terminate the pregnancy, and the timing of labour and delivery in the second and third trimesters, particularly in asymptomatic or mild maternal SARS-CoV-2 infection. These questions can only be answered by further studies, but a relevant multidisciplinary team must also be aware of these risks associated with possibly fatal consequences.

\section{ABBREVIATIONS}

COVID-19, Coronavirus disease 2019; SARS-CoV-2, Acute Respiratory Syndrome Coronavirus 2; CTG, Cardiotocography; US, Ultrasound; BPD, Biparietal diameter; HC, Head circumference; AC, Abdominal circumference; FL, Femur length; EFW, Estimated foetal weigh; UA-PI, Umbilical artery-pulsatility index; MCA-PI, Middle cerebral artery-pulsatility index; CPR, Cerebroplacental 
ratio; $\mathrm{BP}$, Blood pressure; $\mathrm{HR}$, Heart rate; $\mathrm{BT}$, Body temperature; SpO2, Oxygen saturation; WBC, White blood cells; Hb, Haemoglobin; Hct, Haematocrit; PLT, Platelets; CRP - C-reactive protein; RT-PCR, Real-time polymerase chain reaction; CD-68, Cluster of differentiation 68; ACE2, Angiotensin-converting enzyme 2; FIRS, Foetal inflammatory response syndrome; MIS-C, Multisystem inflammatory disorder in children.

Author contributions: KB: manuscript writing, figures; JS: prenatal diagnosis and manuscript revision; TP, TR, EKo: literature search; JM, LP: histopathological analysis and manuscript revision; EKu: manuscript revision and approval.

Conflict of interest statement: The authors state that there are no conflicts of interest regarding the publication of this article.

\section{REFERENCES}

1. Delahoy MJ, Whitaker M, O'Halloran A, Chai SJ, Kirley PD, Alden N, Kawasaki B, Meek J, Yousey-Hindes K, Anderson EJ, Openo KP, Monroe ML, Ryan PA, Fox K, Kim S, Lynfield R, Siebman S, Davis SS, Sosin DM, Barney G, Muse A, Bennett NM, Felsen CB, Billing LM, Shiltz J, Sutton M, West N, Schaffner W, Talbot HK, George A, Spencer M, Ellington S, Galang RR, Gilboa SM, Tong VT, Piasecki A, Brammer L, Fry AM, Hall AJ, Wortham JM, Kim L, Garg S, COVIDNET Surveillance Team. Characteristics and Maternal and Birth Outcomes of Hospitalized Pregnant Women with LaboratoryConfirmed COVID-19 - COVID-NET, 13 States, March 1-August 22 2020. MMWR Morb Mortal Wkly Rep 2020;69:1347-54. doi: 10.15585/ mmwr.mm6938e1

2. Panagiotakopoulos L, Myers TR, Gee J, Lipkind HS, Kharbanda EO, Ryan DS, Williams JT, Naleway AL, Klein NP, Hambidge SJ, Jacobsen SJ, Glanz JM, Jackson LA, Shimabukuro TT, Weintraub ES. SARS CoV-2 Infection Among Hospitalized Pregnant Women: Reasons for Admission and Pregnancy Characteristics - Eight U.S. Health Care Centers, March 1-May 30, 2020. MMWR Morb Mortal Wkly Rep 2020;69:1355-9. doi: 10.15585/mmwr.mm6938e2

3. Poisson TM, Pierone G Jr. Placental pathology and fetal demise at 35 weeks of gestation in a woman with SARS-CoV-2 infection: A case report. Case Rep Womens Health 2021;30:e00289. doi: 10.1016/j. crwh.2021.e00289
4. Zhang H, Wang CY, Zhou P, Yue H, Du R. Histopathologic Changes and SARS-CoV-2 Immunostaining in the Lung of a Patient With COVID-19. Ann Intern Med 2020;173(4):324. doi: 10.7326/L20-0895

5. Sauter JL, Baine MK, Butnor KJ, Buonocore DJ, Chang JC, Jungbluth AA, Szabolcs MJ, Morjaria S, Mount SL, Rekhtman N, Selbs E, Sheng ZM, Xiao Y, Kleiner DE, Pittaluga S, Taubenberger JK, Rapkiewicz AV, Travis WD. Insights into pathogenesis of fatal COVID-19 pneumonia from histopathology with immunohistochemical and viral RNA studies. Histopathology 2020;77(6):915-25. doi: 10.1111/his.14201

6. Gengler C, Dubruc E, Favre G, Greub G, de Leval L, Baud D. SARSCoV-2 ACE-receptor detection in the placenta throughout pregnancy. Clin Microbiol Infect 2021;27(3):489-90. doi: 10.1016/j. cmi.2020.09.049

7. Vivanti AJ, Vauloup-Fellous C, Prevot S, Zupan V, Suffee C, Do Cao J, Benachi A, De Luca D. Transplacental transmission of SARS-CoV-2 infection. Nat Commun 2020;11(1):3572. doi: 10.1038/s41467-02017436-6

8. Menter T, Mertz KD, Jiang S, Chen H, Monod C, Tzankov A, Waldvogel S, Schulzke SM, Hösli I, Bruder E. Placental Pathology Findings during and after SARS-CoV-2 Infection: Features of Villitis and Malperfusion. Pathobiology 2021;88(1):69-77. doi: 10.1159/000511324

9. Kotlyar AM, Grechukhina O, Chen A, Popkhadze S, Grimshaw A, Tal O, Taylor HS, Tal R Vertical transmission of COVID-19: a systematic review and meta-analysis. Am J Obstet Gynecol 2021;224(1):35-53. e3. doi: 10.1016/j.ajog.2020.07.049

10. McCarty KL, Tucker M, Lee G, Pandey V. Fetal Inflammatory Response Syndrome Associated With Maternal SARS-CoV-2 Infection. 2020 Oct 29;e2020010132. [Epub ahead of print] doi: 10.1542/peds.2020010132

11. Diriba K, Awulachew E, Getu E. The effect of coronavirus infection (SARS-CoV-2, MERS-CoV, and SARS-CoV) during pregnancy and the possibility of vertical maternal-fetal transmission: a systematic review and meta-analysis. Eur J Med Res 2020;25(1):39. doi: 10.1186/ s40001-020-00439-w

12. Dong L, Tian J, He S, Zhu C, Wang J, Liu C, Yang J. Possible vertical transmission of SARS-CoV-2 from an infected mother to her newborn. JAMA 2020;323(18):1846-8. doi: 10.1001/jama.2020.4621

13. Rafferty MS, Burrows $H$, Joseph JP, Leveille J, Nihtianova S, Amirian ES. Multisystem inflammatory syndrome in children (MIS-C) and the coronavirus pandemic: Current knowledge and implications for public health. J Infect Public Health 2021;14(4):484-94. doi: 10.1016/j. jiph.2021.01.008

14. Adhikari EH, Moreno W, Zofkie AC, MacDonald L, Mclntire DD Collins RRJ, Spong CY. Pregnancy Outcomes Among Women With and Without Severe Acute Respiratory Syndrome Coronavirus 2 Infection. JAMA Netw Open 2020;3(11):e2029256. doi: 10.1001/ jamanetworkopen.2020.29256 